Proceedings of 7th European Conference on Artificial Life (ECAL2003), W. Banzhaf, T. Christaller, and J. Ziegler (Eds.)

(held at Dortmund, Germany, September 14-17, 2003)

\title{
Meta-Evolutionary Game Dynamics for Mathematical Modelling of Rules Dynamics
}

\author{
Takashi Hashimoto ${ }^{1,2}$ and Yuya Kumagai ${ }^{1}$ \\ 1 School of Knowledge Science, \\ Japan Advanced Institute of Science and Technology (JAIST) \\ 1-1, Tatsunokuchi, Ishikawa, 923-1292, JAPAN \\ hash@jaist.ac.jp http://www.jaist.ac.jp/ ^hash/ \\ 2 Language Evolution and Computation Research Unit, \\ School of Philosophy, Psychology and Language Sciences, \\ University of Edinburgh
}

\begin{abstract}
This paper proposes an evolutionary-game-theory model, called meta-evolutionary game dynamics, for studying the dynamics of rules and individual behaviour. Although there are two game theoretical views of rules, i.e., seeing rules as game forms and as equilibria of games, endogenous changes of rules are not modelled effectively by either of these two views. We introduce a model for integrating the two views, in which the interaction rules of replicator equations change dynamically. Computer simulations of an example of the model that include mutation and extinction of both strategies and games show (1) an intermittent change of strategy distributions, (2) a continual transition from a dominant strategy to another, and (3) metastable states distinct from the Nash equilibria. We discuss the notion of evolutionary stability of games and some natural examples showing rule dynamics. We conclude that meta-evolutionary game dynamics enables the study of the endogenous dynamic of rules. Our model contributes, therefore, the development of game theory approach to the dynamics of rules.
\end{abstract}

Keywords: Dynamics of rules; Mathematical modelling; Meta-evolutionary game dynamics; Replicator equations; Evolutionary stable games

\section{Introduction}

There are certain social "rules", such as institutions, norms, ethics, conventions, laws, and languages. Not all of these rules are given a priori, but many rules emerge spontaneously from our activities. These spontaneous rules constructed by human activities undergo change during the course of history. The changes of the rules are induced by interactions between the rules and the individual activities regulated by the rules.

\subsection{Background: Game Theory Views of Rules}

Conventions, norms, and institutions, which we refer to as "rules" in this paper, and their dynamics have been studied in terms of game theory and evolutionary game theory $[1-5]$. 
There are two views of rules in the framework of game theory [5]. ${ }^{3}$ One view is to see rules as game forms of games, and individuals' actions as moves or strategies of the game players $[1,2]$. In this view, individual behaviour is regulated via the payoffs in the games. Rules should be changed from the outside of the system. Rule changes are represented by, for instance, the change of payoff matrices of the existing games or the addition of new strategies.

The other view considers rules as equilibria of a game [3-5]. In this view, individuals following particular rules are players, and properties of the rules are strategies of the players at the equilibria. Exogenous features that are common to all players, such as environmental factors and laws, are specified as a game form including payoff functions. The actually playable equilibria are thought of as rules established and enforced.

This view may include the evolutionary game framework [6], in which the population or the proportion of individuals taking particular strategies changes as a result of games played. Although it seems in this framework that there is formation of a rule by means of transition from a non-equilibrium state to an equilibrium state, however, the process is actually a selection of one equilibrium out of several prescribed ones. Furthermore, the system no longer changes once it attains an equilibrium state, unless perturbations, like invasions of new strategies or changes of the payoff matrix, are given exogenously. Thus, the spontaneous change of rules cannot be treated.

\subsection{Integration of Two Views}

The common inclinations in both views are that established rules are static and do not change endogenously. As such, these views fail to grasp the fact that rules are dynamic in nature and undergo transition through individuals' activities. We need a new view and a mathematical tool to study the dynamic aspect of rules, one that recognizes that rules and the individuals' behaviour interact with each other and change concurrently. In this paper, we develop a mathematical model of the interaction between rules and individual activities, to expand evolutionary game theory to understand the endogenous dynamics of social structures.

The new view proposed here is an integrated version of the two views above. We basically express initial rules in a society by game forms. Multiple games, which are introduced explicitly, have their own weight factors representing the extent of importance of the rules. Each player plays all games with one strategy and gains weighted payoff from each game. The population of strategies varies in time through the payoffs. In addition, the weights of the games change, depending on the payoffs and the population of strategies. The change of the weights is governed by another rule, called a meta-rule.

The weighted sum of all games can be considered as indicating an entire rule in the society. Accordingly, this entire rule shifts continuously, rather than dis-

\footnotetext{
${ }^{3}$ Aoki [5] lists three views of institutions, the third one of which is 'institutions as specific players of games'. Since this view is not pursued so much, we do not deal with it.
} 
cretely as in transition among equilibria. The rules and configurations of strategies may also stay at an equilibrium, which is a stable rule in both senses described in the previous subsection.

The situation abstracted here may be acceptable when we suppose multinational firms engaged in business activities in multiple markets. Each market has its own scale and degree of importance in the world economy. The basic strategy of a firm is thought of as the same for all markets, even though the firm can adjust its tactics for each market. Firms change their shares according to the benefits obtained at all markets. The scale and the importance of each market also change in time with the activities of the firms.

\subsection{Meta-Rule and Hierarchy of Rules Plasticity}

One might ask what a meta-rule represents. It is a principle regarded as more basic than an objective rule to be modelled, and therefore it is exogenous. In the above example, it is the basic principle of the market mechanism, such that a profitable market is more importance in the world economic system than an unprofitable market. The meta-rule should be appropriately set for an objective system. For instance, it may be the happiness of the majority for a utilitarian system, the diversity of individual opinions for a democratic system, or sportsmanship in sports.

Such a principle, of course, may change in time, or be replaced by a new principle. Thus, we might consider introducing a meta-meta-rule to capture the change of the principle. Logically speaking, the chain of meta-meta-- -rules may cause a problem of infinite regression.

However, we allow a hierarchy of rules plasticity. We suppose that the rules are classified in terms of their variability. As exemplified above, a meta-rule are deemed more universal and invariable than rules at a focal level. For example, in the legal system of a country, the constitution hardly changes, criminal laws can be amended under the constitution, civil laws are more changeable, and so on. In this paper, we assume that the change of rules in the lower classes of the plasticity hierarchy can be neglected. By introducing an invariable metarule, we confine the dynamics within two levels, the rules and the behaviour of individuals.

\section{Meta-Evolutionary Game Dynamics}

In this section, we formalize the views proposed in $\S 1.2$ to model dynamic change of rules. It is an extension of replicator dynamics [7] to include multiple games with variable weights. We call our formalization meta-evolutionary game dynamics.

Suppose $N$ different strategies and the population share of individuals taking the $i$-th strategy denoted by $x_{i}$, where $\sum_{i=1}^{N} x_{i}=1$. The replicator system is

$$
\dot{x}_{i}=\left(u_{i}-\bar{u}\right) x_{i},
$$


where $u_{i}$ is the payoff of $i$-th strategy and $\bar{u}=\sum_{i=1}^{N} x_{i} u_{i}$ is the average of payoffs over all individuals. This equation implies that the share of the $i$-th strategy grows or shrinks in proportion to the difference between its payoff and the average payoff.

We introduce here $M$ multiple games with weighting factors. The weight of the $g$-th game is expressed by $w^{g}$, where $\sum_{g=1}^{M} w^{g}=1$. All individuals play all games simultaneously with their own strategies. Thus, the growth rate of the share of each strategy consists of the weighted sum of the payoffs at each game. Accordingly, the time evolution of the share is given by

$$
\dot{x}_{i}=\sum_{g=1}^{M} w^{g}\left(u_{i}^{g}-\bar{u}^{g}\right) x_{i},
$$

where $u_{i}^{g}$ and $\bar{u}^{g}=\sum_{i=1}^{N} x_{i} u_{i}^{g}$ are the payoff of the $i$-th strategy and the average payoff at the game $g$, respectively. We refer to this equation as the weighted replicator equation.

We assume that each game is evaluated in terms of certain principles or criteria, i.e., a meta-rule in our terminology. We also assume the weight changes according to the evaluation. It is further postulated that the higher a game is evaluated above average, the more the weight of the game grows. Thus, as with the dynamics of strategies, the dynamics of weights is described by replicator type equation (1), by introducing the evaluation $\lambda^{g}$ of the game $g$,

$$
\dot{w}^{g}=\frac{1}{\tau}\left(\lambda^{g}-\bar{\lambda}\right) w^{g}
$$

where $\tau$ represents the time constant associated with the rate of change relative to the change of strategy shares, and $\bar{\lambda}=\sum_{g=1}^{M} w^{g} \lambda^{g}$ is the weighted average of the evaluation over all games. We refer to the whole system of equations (2) and (3), together with the definition of the evaluation function, which will be presented later, as the meta-evolutionary game dynamics.

A meta-rule regulating the dynamics of games is introduced as the form of the evaluation function. Basically, the meta-rule is considered as the function of the population shares and the payoffs of all strategies, namely, $\lambda^{g}=\lambda^{g}\left(\boldsymbol{x}, \boldsymbol{u}^{g}\right)$, where $\boldsymbol{x}=\left(x_{1}, x_{2}, \cdots, x_{N}\right)$ is a vector of population shares called the strategy profile and $\boldsymbol{u}^{g}(\boldsymbol{x})=\left(u_{1}^{g}(\boldsymbol{x}), u_{2}^{g}(\boldsymbol{x}), \cdots, u_{N}^{g}(\boldsymbol{x})\right)$ is a vector of the payoffs at the game $g$.

We can define various evaluation schemes according to the objects to be modelled. For example, we can evaluate any single stock market based on the average profit it provides to all who invest in it. In the same way, we can evaluate any single game based on the average payoff it provides to all who play it. In this case, it is defined as $\lambda_{\mathrm{A}}^{g}\left(\boldsymbol{x}, \boldsymbol{u}^{g}\right)=\left\langle u_{i}^{g}(\boldsymbol{x})\right\rangle$, where $\langle\cdot\rangle$ means the average over all strategies. In this scheme, a game that gives large payoff to the players on average raises its weight. Another example is the inverse of variance, $\lambda_{\mathrm{IV}}^{g}\left(\boldsymbol{x}, \boldsymbol{u}^{g}\right)=\left(\left\langle u_{i}^{g}(\boldsymbol{x})-\left\langle u_{i}^{g}(\boldsymbol{x})\right\rangle\right\rangle\right)^{-1}$. This evaluation function represents a situ- 
ation in which equality or impartiality acquires importance as in a democratic society. ${ }^{4}$

We suppose that the state of a societal rule is characterized by the weighted average of the component rules. Then, the total game is defined as

$$
G=\sum_{g=1}^{M} w^{g} A^{g}
$$

where $A^{g}(g=1, \cdots, M)$ stands for the payoff matrices of a component game $g$. Note that this matrix is not constant, since each weight $w^{g}$ changes in time according to Eq. (3). Using the total game $G$, the population dynamics, namely, the weighted replicator dynamics, Eq. (2), is reduced to the matrix form,

$$
\dot{\boldsymbol{x}}=(G(t) \boldsymbol{x}-\boldsymbol{x} \cdot G(t) \boldsymbol{x}) \boldsymbol{x},
$$

where the operator ' '' is the inner product between vectors. This formalization clearly shows that meta-evolutionary game dynamics is a direct development of the replicator equation into one with a time-changing interaction matrix. ${ }^{5}$

\section{Model}

\subsection{Specification of Simulation Model}

We specify a particular model of meta-evolutionary game dynamics for $2 \times 2$ symmetric game matrices and individuals characterized by mixed strategies. ${ }^{6}$ In this model, the basic equations (2) and (3) were reformalized into difference equations. In addition, mutation and extinction of both the strategies and the games were introduced as follows. The values in parentheses were used in the following simulations described in $\S 4$.

Games: All games are $2 \times 2$ symmetric matrices with elements $a_{i j} \in[-1,1]$.

Strategies: We adopt a mixed strategy $\left(s_{1}, s_{2}\right)$ for an individual, where $s_{1}, s_{2} \in$ $[0,1]$, and $s_{1}+s_{2}=1$. (We use 11 strategies $\left.s_{1}=(0.0,0.1, \cdots, 1.0)\right)$.

Mutation of strategies: The ratio $\mu_{s}(=0.003)$ of the share of each strategy is transferred to the other strategies or to a new strategy at every step.

Mutation of games: The ratio $\mu_{g}(=0.003)$ of the weight of a randomly selected game is transferred to a new game at every step. Each element of the new game is shifted by a random number of normal distribution with the average 1 and the variance 0 from the original game.

\footnotetext{
${ }^{4}$ The payoff matrices of all games should be appropriately normalized or limited for each evaluation function.

5 The meta-evolutionary game dynamics may be represented as continuous-time infinite dynamic games or differential games [8]. The cost function to be maximized in that framework, however, is defined as a time integration of payoff at a point in time or at one-shot game. Furthermore, following the definition in [8], the payoff function at a point in time does not include payoff values at previous times.

${ }^{6}$ In the usual replicator dynamics, an individual is thought to be taking a pure strategy, and the strategy profile can be interpreted as a mixed strategy.
} 
Extinction of strategies: The strategies with the share $x_{i}<\theta_{s}\left(=10^{-7}\right)$ are removed.

Extinction of games: The games with the weight $w^{g}<\theta_{g}\left(=10^{-7}\right)$ are removed.

\subsection{Categorization of $2 \times 2$ Game Matrix}

Followings are the normalization and the categorization of game matrices. Since the replicator dynamics is invariant under a local shift of payoff [6], given a $2 \times 2$ payoff matrix $A=\left(\begin{array}{ll}a & b \\ c & d\end{array}\right)$, then we can transform the payoff matrix without loss of generality to

$$
A^{\prime}=\left(\begin{array}{cc}
a-c & 0 \\
0 & d-b
\end{array}\right) \equiv\left(\begin{array}{ll}
\alpha & 0 \\
0 & \beta
\end{array}\right) .
$$

Under this transformation, the set of Nash equilibria is invariant. We use the $\alpha-\beta$ space to view the motion of games. The $\alpha-\beta$ space is divided into four categories in terms of properties of the set $\Theta^{N E}$ of Nash equilibria and the convergence point $\hat{\boldsymbol{x}}$ of the replicator dynamics [6]. Let the strategies for $2 \times 2$ game be $\boldsymbol{e}_{0}=(1,0), \boldsymbol{e}_{1}=(0,1)$, and $\boldsymbol{p}=\left(\frac{\beta}{\alpha+\beta} \frac{\alpha}{\alpha+\beta}\right)$. Then the four categories are defined as follows:

Category I For a game with $\alpha<0$ and $\beta>0, \Theta^{N E}=\left\{\boldsymbol{e}_{0}\right\}$ and $\hat{\boldsymbol{x}}=\boldsymbol{e}_{0}$.

Category II For $\alpha>0$ and $\beta>0, \Theta^{N E}=\left\{\boldsymbol{e}_{0}, \boldsymbol{p}, \boldsymbol{e}_{1}\right\}$. If the initial state $\boldsymbol{x}_{0}$ is $\boldsymbol{e}_{0}<\boldsymbol{x}_{0}<\boldsymbol{p}$, then $\hat{\boldsymbol{x}}=\boldsymbol{e}_{0}$. If $\boldsymbol{p}<\boldsymbol{x}_{0}<\boldsymbol{e}_{1}$, then $\hat{\boldsymbol{x}}=\boldsymbol{e}_{1}$.

Category III For $\alpha<0$ and $\beta<0, \Theta^{N E}=\{\boldsymbol{p}\}$ and $\hat{\boldsymbol{x}}=\boldsymbol{p}$.

Category IV For $\alpha>0$ and $\beta<0, \Theta^{N E}=\left\{\boldsymbol{e}_{1}\right\}$ and $\hat{\boldsymbol{x}}=\boldsymbol{e}_{1}$.

\section{Simulation Results of Average-Type Meta-Rule}

As a simple example of the meta-rule, we examined the average type meta-rule,

$$
\lambda_{\mathrm{A}}^{g}\left(\boldsymbol{x}, \boldsymbol{u}^{g}(\boldsymbol{x})\right)=\frac{1}{N} \sum_{i=1}^{N} u_{i}^{g}(\boldsymbol{x}) .
$$

This evaluation function formalizes a meta-rule that selects the rules that gives the maximum utility to individuals on average.

An example of the time evolution of population share for each strategy is shown in Fig.1. In this and the following results, only one game whose elements are all 0.0 exists and four strategies are selected using random numbers with uniform distribution at the initial state. The distribution of share intermittently changes in time. Moreover, there occasionally arise the transitions in a dominant strategy sometimes. 


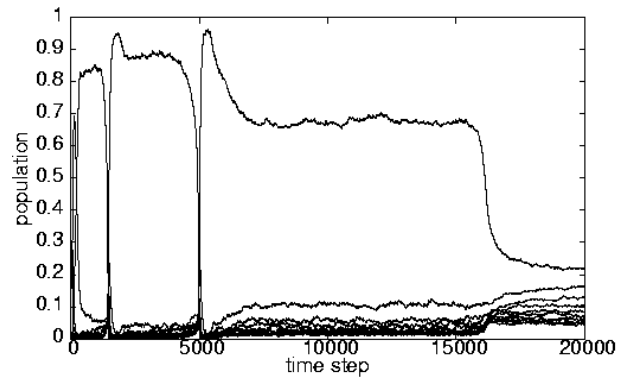

Fig. 1. The time evolution of the share of strategies for the averagetype meta-rule. The $x$-axis is the time step and the $y$-axis is the share. The time constant $\tau=4.0$. All 11 lines for the strategies are drawn. We can observe the changes of the distribution and transitions of the dominant strategies.

To see the movement of games, we depict in Fig.2 the change of the elements $\alpha$ and $\beta$ of the normalized total game. The large changes in the population share correspond with the large changes in the total game. The drastic change of distribution of strategies and the change of dominant strategies are caused by the shift of the total game between or inside the categories. In the $\alpha-\beta$ plane, the total game starts from the origin, moves among the categories, and finally comes back toward the origin.

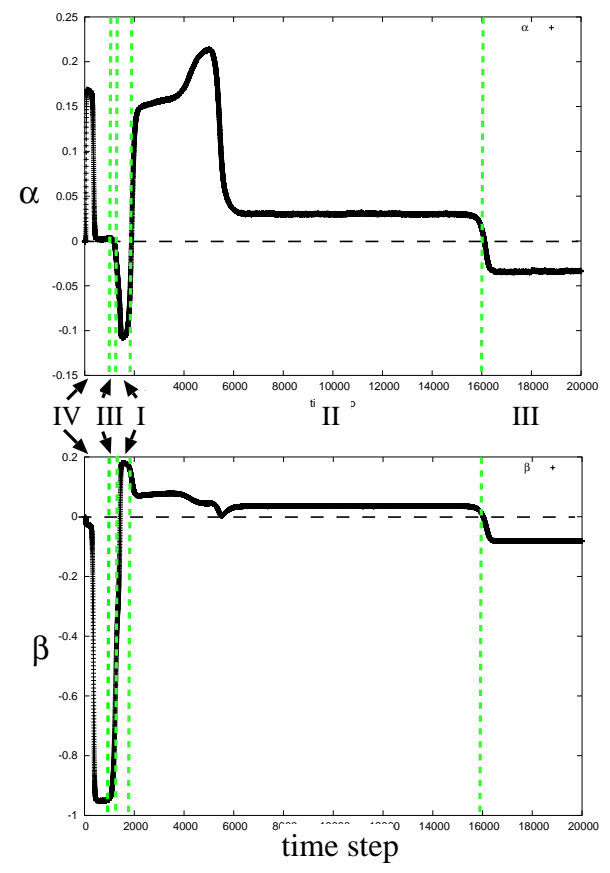

Fig. 2. The time evolution of $\alpha$ (upper) and $\beta$ (lower) of the total game. The $x$-axis denotes the time step. Categories I IV (introduced in $\S 3.2$ ) and the line dividing them are indicated.

The usual replicator dynamics with a $2 \times 2$ matrix has a stable equilibrium at one of two pure strategies in each of the categories I, II, and IV. However, the actual distribution of the strategies in meta-evolutionary dynamics does not converge to these Nash equilibria of the total game, as shown in Fig.3. For categories I, II, and IV, this misconvergence is induced by the perturbation caused by strategy mutation. Therefore, the difference between the actual distribution and the equilibria is small.

For category III, the mutation also affects the misconveregence, but it seems to be more than a perturbation. The system presumably diverges in a disparate direction from the equilibrium under the appropriate mutation rate. ${ }^{7}$ In the ideal (without perturbations) situation,

\footnotetext{
${ }^{7}$ If the mutation rate is too large, the structure of the system is destroyed. Thus there is no remarkable dynamics as described here, and the difference between the equilibrium and the actual stationary point is just proportional to the mutation rate.
} 
the distribution of strategies would converge to the Nash equilibrium of the total game. With perturbations, however, the distribution of strategies converges to a metastable point, which is different from the equilibrium point. To understand the mechanism that causes the two points to be different, we should analyse each game not only the total game.

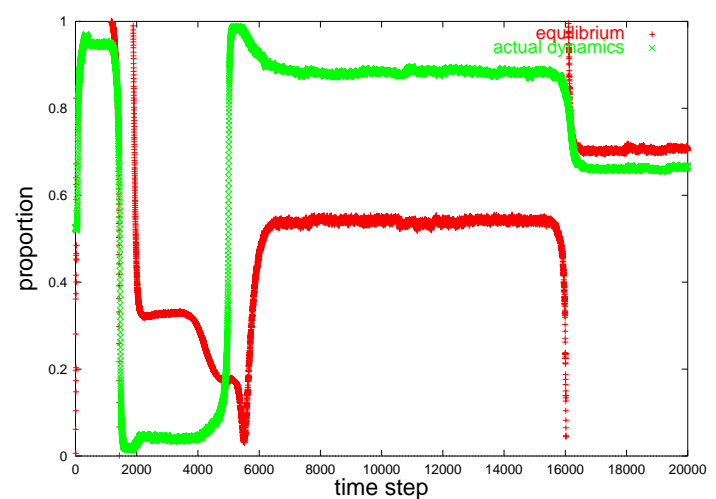

Fig. 3. The comparison of time evolutions of the actual dynamics $(\times$, gray) and the equilibrium point of the total game $(+$, black). The $x$-axis is the time step and the $y$-axis is the proportion of the first pure strategy. The symbol $\times$ 's are for the actual dynamics, $\sum_{i} x_{i} s_{1}$, and + 's are that of the theoretically calculated equilibrium of the total game.

\section{Discussion}

\subsection{Evolutionary Stable Game}

In meta-evolutionary game dynamics, the weights of games change according to Eq. (3). If a strategy profile is fixed, the games reach an equilibrium under the profile. Therefore, the evolutionary stability of games is formalized as the straightforward extension of the evolutionary stable strategy in evolutionary game theory [9].

Suppose a strategy profile $\boldsymbol{x}$. When a game $g$ (possibly the total game) satisfies

$$
\lambda^{g}\left(\boldsymbol{x}, \boldsymbol{u}^{g}\right)>\lambda^{g^{\prime}}\left(\boldsymbol{x}, \boldsymbol{u}^{g^{\prime}}\right)
$$

for any $g^{\prime}$ under the profile $\boldsymbol{x}$, the game $g$ is stable under the invasion of any game. Furthermore, if the strategy profile $\boldsymbol{x}$ is the evolutionary stable strategy, there is no incentive for both the game and the strategy to change. "Evolutionary stable strategy" satisfies

$$
\boldsymbol{x} \cdot A^{g}(\epsilon \boldsymbol{y}+(1-\epsilon) \boldsymbol{x})>\boldsymbol{y} \cdot A^{g}(\epsilon \boldsymbol{y}+(1-\epsilon) \boldsymbol{x})
$$

for sufficiently small $\epsilon>0$ and for any strategy profile $\boldsymbol{y}$, where $A^{g}$ is the payoff matrix of the game $g$. Thus, the system is at an equilibrium with respect to both the game and the strategy. We call the game $g$ and the strategy profile $\boldsymbol{x}$ satisfying Eq. (8) and (9) an evolutionary stable game (ESG). The ESG of the average-type meta-rule under the specifications of $\S 3$ is $G^{E S G}=\left(\begin{array}{ll}1 & 1 \\ 1 & 1\end{array}\right)$. Therefore, the total game converges to the origin of the $\alpha$ - $\beta$ space. 
We found that, in the simulation of the average-type meta-rule with mutation and extinction, the meta-evolutionary game dynamics results in a metastable state distinct from the Nash equilibria. In general, for a system in which ESG is definable and mutation is introduced, since the strategy profile changes slowly around Nash equilibria, we infer the existence of a metastable state different from Nash equilibria.

We have also examined a system with the variance-type meta-rule

$$
\lambda_{\mathrm{V}}^{g}\left(\boldsymbol{x}, \boldsymbol{u}^{g}(\boldsymbol{x})\right)=\frac{1}{N} \sum_{i=1}^{N}\left(u_{i}^{g}(\boldsymbol{x})-\frac{1}{N} \sum_{i=1}^{N} u_{i}^{g}(\boldsymbol{x})\right) .
$$

Since this evaluation function gives a high weighting factor to a game in which the variance of the payoff of strategies is large, the difference of payoffs between winners and losers increases and one strategy tends to dominate. In such a situation, the other strategies decrease their population, and sooner or later they become extinct. When they disappear, there remains only one strategy. In such a situation, the variance also disappears and, therefore, the evaluation of such a game decreases drastically. Accordingly, ESG is not defined for this meta-rule and the games and the strategies tend to be itinerant.

\subsection{Various Systems with Dynamics of Rules}

Natural examples in which rules of a system change with time are ubiquitous. Almost all adaptive systems show dynamics of rules. The brain has a hierarchical structure, i.e., upper levels regulate lower levels to some extent, the dynamics at the upper level induce change of the rule of dynamics at the lower level [10]. Cognitive development is a process of rewriting rules for representation [11]. Biological evolution is the transition of functions that determine characteristics of biological systems [12].

In this paper, we treat social systems such that the behaviour of elements at the micro or lower level not only is governed by a rule, the macro or upper level, but also can change the rule. In other words, the system has a dynamic interaction loop between the two levels. Language is another representative what having such dynamics. Grammars and lexicons of a language are kinds of rules that the users of the language have produced and followed. Such rules change temporally to a greater or lesser extent. Changes are brought about by the users' activities, especially writing and speaking the language. Unused, or dead, languages are indisputably invariable.

It is difficult to treat the dynamics of rules mathematically and generically. In the theory of dynamical systems, state changes of the system are governed by fixed functions. The change of functions is beyond the scope of the standard dynamical systems theory. Recently, some studies in iterated functional systems, skew product, and functional shift [13], have considered change of functions. To understand the dynamics of rules, we need to develop a mathematical framework for modelling the dynamics, as well as describing them empirically. 


\section{Conclusion}

We conclude that the meta-evolutionary game dynamics proposed in the present paper is useful for studying the dynamic change of rules in which the interaction loop between individual behaviour and the state of rules matters. The endogenous change of rules cannot be studied effectively by either the game-form view or the equilibrium view. Such interesting dynamics, intermittent changes of the distribution of strategies, a continual drastic transition of the dominant strategies, and metastable states different from the Nash equilibria are not seen in the usual low-dimension replicator dynamics. Further study of meta-evolutionary game dynamics will contribute to an understanding of rule dynamics from the viewpoint of game theory. We must clarify foremost how such dynamics occur by looking closely at the dynamics of both the total game and of component games. Moreover, applicability of meta-evolutionary game dynamics to the empirical investigation of the formation of norms and institutions should be pursued.

Acknowledgement The authors thank Prof. DiGiovanni for his critical reading of the manuscript. This work is partly supported by the Research Fellowship Program of Canon Foundation in Europe, and by a Grant-in-Aid for Scientific Research (No.12780269 and No.15700183) from the Ministry of Education, Culture, Sports, Science and Technology of Japan and by Japan Society for the Promotion of Science.

\section{References}

1. North, D. C.: Institutions, Institutional Change and Economic Performance. Cambridge University Press (1990)

2. Hurwicz, L.: Institutions as Families of Game Forms. Japanese Economic Review, 47 (1996) 113-32

3. Lewis, D.: Convention. Harvard University Press (1969)

4. Young, H. P.: Individual Strategy and Social Structure - An Evolutionary Theory of Institutions. Princeton University Press (1998)

5. Aoki, M.: Toward a Comparative Institutional Analysis. The MIT Press (1996)

6. Weibull, J. W.: Evolutionary Game Theory. MIT Press (1995)

7. Taylor, P., Jonker, L.,: Evolutionary Stable Strategies and Game Dynamics. Mathematical Biosciences 40, (1978) 145-156.

8. Basar, T., Olsder, G. J.: Dynamic Noncooperative Game Theory (2nd Edition). Academic Press (1995)

9. Maynard-Smith, J.: Evolution and the Theory of Games. Cambridge University Press (1982)

10. Tsuda, I.: Toward an interpretation of dynamic neural activity in terms of chaotic dynamical systems. Behavioral and Brain Sciences 24 (2001) 793-847

11. Karmiloff-Smith, A.: Beyond Modularity - A Developmental Perspective on Cognitive Science. The MIT Press (1992)

12. Maynard-Smith, J., Szathmary, E., The Major Transitions in Evolution, Oxford University Press (1995)

13. Namikawa, J., Hashimoto, T.: Dynamics and computation in functional shifts. Submitted to Nonlinearity 